\title{
DDX3X deficiency alleviates LPS-induced H9c2 cardiomyocytes pyroptosis by suppressing activation of NLRP3 inflammasome
}

\author{
DANDAN FENG ${ }^{1}$, LIANG GUO ${ }^{2}$, JING LIU $^{3}$, YUNXUAN SONG ${ }^{4}$, XIUYUAN MA ${ }^{1}$, \\ HAIYANG HU ${ }^{1}$, JU LIU ${ }^{3}$ and ENKUI HAO ${ }^{1}$ \\ ${ }^{1}$ Department of Cardiology, Shandong Provincial Qianfoshan Hospital, Cheeloo College of Medicine, \\ Shandong University, Jinan, Shandong 250012; ${ }^{2}$ Department of Anesthesiology, \\ The First Affiliated Hospital of Shandong First Medical University and Shandong Provincial Qianfoshan Hospital; \\ ${ }^{3}$ Laboratory of Microvascular Medicine, Medical Research Center, The First Affiliated Hospital of \\ Shandong First Medical University and Shandong Provincial Qianfoshan Hospital; ${ }^{4}$ Department of Cardiology, \\ Shandong Provincial Qianfoshan Hospital, Shandong First Medical University, Jinan, Shandong 250014, P.R. China
}

Received February 16, 2021; Accepted August 18, 2021

DOI: $10.3892 /$ etm.2021.10825

\begin{abstract}
Increasing evidence suggest that NOD-like receptor protein 3 (NLRP3) inflammasome-mediated pyroptosis may be the underlying pathological mechanism of sepsis-induced cardiomyopathy. DDX3X, an ATP-dependent RNA helicase, plays a vital role in the formation of the NLRP3 inflammasome by directly interacting with cytoplasmic NLRP3. However, whether DDX3X has a direct impact on lipopolysaccharide (LPS)-induced cardiomyocyte injury by regulating NLRP3 inflammasome assembly remains unclear. The present study aimed to investigate the role of DDX3X in the activation of the NLRP3 inflammasome and determine the molecular mechanism of DDX3X action in LPS-induced pyroptosis in H9c2 cardiomyocytes. H9c2 cardiomyocytes were treated with LPS to simulate sepsis in vitro. The results demonstrated that LPS stimulation upregulated DDX3X expression in $\mathrm{H} 9 \mathrm{c} 2$ cardiomyocytes. Furthermore, $D d x 3 x$ knockdown significantly attenuated pyroptosis and cell injury in LPS-treated H9c2 cells by suppressing NLRP3 inflammasome activation. Taken together, these results suggest that DDX3X is involved in LPS-induced cardiomyocyte pyroptosis, and DDX3X deficiency mitigates cardiomyocyte damage induced by LPS treatment.
\end{abstract}

Correspondence to: Dr Enkui Hao, Department of Cardiology, Shandong Provincial Qianfoshan Hospital, Cheeloo College of Medicine, Shandong University, 16766 Jingshi Road, Jinan, Shandong 250012, P.R. China

E-mail: haoenkui@sdu.edu.cn

Key words: DDX3X, NOD-like receptor protein 3 inflammasome, pyroptosis, reactive oxygen species, lipopolysaccharide-induced cardiomyocyte injury

\section{Introduction}

Sepsis is a clinical syndrome that occurs following infection or injury (1). If not timely and properly controlled, sepsis can develop into systemic inflammatory response syndrome and ultimately result in multiple organ dysfunction syndrome (MODS) (2). Cardiac dysfunction is a severe sepsis-related complication characterized by left ventricular dilatation, decreased ejection fraction and recovery in 7-10 days (3). The molecular mechanisms of cardiac tissue damage during sepsis remain elusive. In previous studies, we examined the role of inflammation, oxidative stress, apoptosis and autophagy in sepsis-related myocardial injury $(4,5)$. Recently, pyroptosis, a specific type of programmed cell death, was reported as a common cause of sepsis-induced tissue damage (6-9). During the development of sepsis, pyroptosis can destroy the integrity of cell membranes, resulting in inflammatory cytokine secretion and augmented inflammatory responses $(10,11)$. Thus, selective suppression of genes and proteins involved in pyroptosis may be a potential therapeutic strategy for sepsis or sepsis-induced cardiomyopathy.

The NOD-like receptor protein 3 (NLRP3) inflammasome is a multiprotein heteromeric complex that detected a variety of danger signals that originate not only from microorganisms but also from metabolic disorders. The assembly of the NLRP3 inflammasome contributes to the self-shearing of caspase-1, leading to the generation of activated caspase-1 fragments. Activated caspase-1 induces the maturation of the proinflammatory cytokines, pro-IL-1 $\beta$ and pro-IL-18. In addition, activated caspase-1 shears gasdermin D (GSDMD); the cleaved $\mathrm{N}$-terminal domain of GSDMD translocates to the plasma membrane and forms pores, thereby facilitating the extracellular secretion of inflammatory cytokines into the circulation system and triggering the classical pathway of pyroptosis (12). Increasing evidence suggest that the NLRP3/caspase-1/GSDMD signaling pathway is involved in several pathophysiological mechanisms, such as innate immunity, myeloid proliferation, tumorigenesis and Alzheimer's disease (13-19). It has also been 
reported that NLRP3 inflammasome activation requires two signals, a priming signal and an activating signal. First, the priming signal provided by pathogen- and danger-associated molecular patterns activates the $\mathrm{NF}-\kappa \mathrm{B}$ signaling pathway and subsequently upregulates the expression levels of NLRP3 and pro-IL-1 $\beta$ (20). The activating signal is then provided by a variety of molecular or cellular events, including reactive oxygen species (ROS) production, ionic flux and lysosomal damage (21). Compared with the latter two models, the ROS model was considered one of the most crucial signaling pathways for the activation of the NLRP3 inflammasome (22). Mechanistically, increased intracellular ROS levels can lead to the detachment of TXNIP from the TXNIP-Trx protein complex, then free TXNIP can bind to NLRP3, resulting in NLRP3 activation and promoting the assembly of the NLRP3 inflammasome (23).

DDX3X, an ATPase/RNA helicase of the DEAD-box family, participates in several RNA metabolic processes (24). This protein is also involved in cell cycle progression, apoptosis, antiviral innate immunity and cancer development (25-28). A recent study demonstrated that DDX3X is crucial for NLRP3 inflammasome assembly due to its direct binding interaction with NLRP3, and $D d x 3 x$ knockdown in peritoneal macrophages suppresses NLRP3 inflammasome activation and reduces pyroptosis (29). The binding site for DDX3X is in the NACHT region of NLRP3, which exerts ATPase activity required for NLRP3 oligomerization following activation. This suggests that DDX3X plays an indispensable role in facilitating the oligomerization of NLRP3 (30-32).

To the best of our knowledge, the role of DDX3X in lipopolysaccharide (LPS)-induced cardiomyocyte stress response has not yet been investigated. Thus, the present study aimed to determine whether DDX3X participates in LPS-induced cardiomyocyte injury by regulating NLRP3 inflammasome formation and subsequent pyroptosis.

\section{Materials and methods}

Cell culture and treatment. The H9c2 rat myocardial cell line was purchased from the National Collection of Authenticated Cell Culture (https://cellbank.org.cn). H9c2 cells were maintained in DMEM (cat. no. 12800017) supplemented with $1.5 \mathrm{~g} / 1 \mathrm{NaHCO}_{3}, 10 \%$ fetal bovine serum (all purchased from Gibco; Thermo Fisher Scientific, Inc.) and $1 \%$ antibiotics (100 U/ml penicillin and $100 \mathrm{mg} / \mathrm{ml}$ streptomycin; Beijing Solarbio Science \& Technology Co., Ltd.), at $37^{\circ} \mathrm{C}$ with $5 \% \mathrm{CO}_{2}$.

LPS was purchased from MedChemExpress (cat. no. HY-D1056) and reconstituted in DMSO. When the cells reached $70 \%$ confluence, LPS $(1 \mu \mathrm{g} / \mathrm{ml})$ was added to the culture medium to mimic sepsis-induced cardiomyocyte pyroptosis in vitro; the control group was treated with DMSO.

Cell transfection. Small interfering (si)RNA against rat $D d x 3 x$ and scramble siRNA were purchased from Shanghai GenePharma Co., Ltd. Scramble siRNA was used as the negative control for siRNA silencing. When $\mathrm{H} 9 \mathrm{c} 2$ cells reached $50-60 \%$ confluence, siRNA was transfected into H9c2 cells using Lipofectamine ${ }^{\circledR} 3000$ (Invitrogen; Thermo Fisher Scientific, Inc.), according to the manufacturer's instructions.
Briefly, 50 nM DDX3X siRNA or scramble siRNA in combination with $5 \mu 1$ of Lipofectamine ${ }^{\circledR} 3000$ was added to each well. Following incubation with serum-free DMEM at $37^{\circ} \mathrm{C}$ for $6 \mathrm{~h}$, the medium was replaced with DMEM containing serum. After additional incubation at $37^{\circ} \mathrm{C}$ for $18 \mathrm{~h}, \mathrm{H} 9 \mathrm{c} 2$ cells were treated with LPS $(1 \mu \mathrm{g} / \mathrm{ml})$. The sense and antisense siRNA sequences are listed in Table I.

Western blotting. Total protein extraction was performed following treatment with LPS for $24 \mathrm{~h}$. RIPA lysis buffer supplemented with $1 \mathrm{mM}$ phenylmethylsulfonyl fluoride (Beyotime Institute of Biotechnology) was added dropwise to each well to lyse the cells. The supernatant of the cell lysates was collected following centrifugation at 11,588 $\mathrm{x}$ g for $15 \mathrm{~min}$ at $4^{\circ} \mathrm{C}$. For secretory protein extraction, cell medium supernatant was collected and centrifuged at $600 \mathrm{x}$ g for $5 \mathrm{~min}$ at $4^{\circ} \mathrm{C}$ and $11,588 \times \mathrm{g}$ for $15 \mathrm{~min}$ at $4^{\circ} \mathrm{C}$. The bicinchoninic acid assay kit (Beyotime Institute of Biotechnology) was used to quantify protein samples, according to the manufacturer's instructions. Equal amounts of protein samples $(30 \mu \mathrm{g})$ were separated by 10, 12.5 and 15\% SDS-PAGE (EpiZyme), transferred onto PVDF membranes and blocked with 5\% non-fat milk at room temperature for $1 \mathrm{~h}$. The membranes were incubated with primary antibodies against rabbit anti-GAPDH (cat. no. 5174; 1:8,000; Cell Signaling Technology, Inc.), rabbit anti-DDX3X (cat. no. 11115-1-AP; 1:1,000; ProteinTech Group, Inc.), rabbit anti-Caspase1/P20/P10 (cat. no. 22915-1-AP; 1:1,500; ProteinTech Group, Inc.), rabbit anti-NLRP3 (cat. no. 19771-1-AP; 1:1,000; ProteinTech Group, Inc.), rabbit anti-IL-1 $\beta$ (cat. no. AF5103; 1:1,000; Affinity Biosciences) and rabbit anti-Cleaved-IL-1 $\beta$ (cat. no. AF4006; 1:1,000; Affinity Biosciences) overnight at $4^{\circ} \mathrm{C}$. GAPDH was used as the internal control. The PVDF membranes were washed three times with Tris-buffered saline with 1\% Tween-20 (Beyotime Institute of Biotechnology) and subsequently incubated with Anti-rabbit IgG, HRP-linked Antibody (cat. no. 7074; 1:10,000; Cell Signaling Technology, Inc.) at room temperature for $1 \mathrm{~h}$. Coomassie blue staining was performed by incubating the gels with Coomassie blue staining solution (Beyotime Institute of Biotechnology) at room temperature for $30 \mathrm{~min}$ and washing them in Coomassie blue eluent (methanol:glacial acetic acid : distilled water $=3: 1: 6$ ) until clear bands appeared. The immunoblots were detected using chemiluminescence reagents (MilliporeSigma) and the blots were scanned using a chemiluminescent analyzer (ProteinSimple). Relative immunoblot intensities were analyzed using ImageJ v1.8.0.112 software (National Institutes of Health).

Reverse transcription-quantitative $(R T-q) P C R$. Following treatment with LPS for $24 \mathrm{~h}, \mathrm{TRIzol}^{\circledR}$ reagent (Invitrogen; Thermo Fisher Scientific, Inc.) was used to extract total RNA from H9c2 cells. Equal amounts of total RNA (1-2 $\mu \mathrm{g})$ were reverse transcribed into cDNA using the HiScript III RT SuperMix for qPCR (+gDNA wiper; Vazyme Biotech Co., Ltd.). The following temperature protocol was used for reverse transcription: $42^{\circ} \mathrm{C}$ for $2 \mathrm{~min}$, followed by $37^{\circ} \mathrm{C}$ for $15 \mathrm{~min}$ and $85^{\circ} \mathrm{C}$ for $5 \mathrm{sec}$. Primer sequences were purchased from BGI (https://www.bgi.com/). qPCR was performed in a 20- $\mu$ l reaction volume consisting of ChamQ Universal SYBR qPCR Master Mix (Vazyme Biotech Co., Ltd.). RT-qPCR was 
Table I. Sense and antisense siRNA sequences.

\begin{tabular}{lc}
\hline Gene & Sequence (5'-3') \\
\hline $\begin{array}{l}\text { DDX3X siRNA } \\
\text { sense strand }\end{array}$ & GGAGGAUUUCUUAUACCAUTT \\
DDX3X siRNA & AUGGUAUAAGAAAUCCUCCTT \\
antisense strand & \\
Control siRNA & UUCUCCGAACGUGUCACGUTT \\
sense strand & \\
Control siRNA & ACGUGACACGUUCGGAGAATT \\
antisense strand &
\end{tabular}

si, small interfering.

performed on a CFX96 Real-Time PCR Detection System (Bio-Rad Laboratories, Inc.). The following thermocycling conditions were used for qPCR: $95^{\circ} \mathrm{C}$ for $30 \mathrm{sec}$, followed by 40 cycles of $95^{\circ} \mathrm{C}$ for $10 \mathrm{sec}, 60^{\circ} \mathrm{C}$ for $30 \mathrm{sec}$ and $65^{\circ} \mathrm{C}$ for $5 \mathrm{sec}$. Relative expression levels were calculated using the $2^{-\Delta \Delta C q}$ method (33) and normalized to the internal reference gene GAPDH. The primer sequences used for qPCR are listed in Table II.

Measurement of caspase-1 activity. Caspase-1 activity was measured using the caspase-1 activity assay kit (Beyotime Institute of Biotechnology), according to the manufacturer's instructions. Absorbance was measured using an enzyme-labeled instrument (BioTek Instruments, Inc.) at a wavelength of $405 \mathrm{~nm}$.

Determination of ROS levels. Following treatment with LPS, the culture medium was removed, and $\mathrm{H} 9 \mathrm{c} 2$ cells were subsequently incubated with $1 \mathrm{ml}$ of serum-free DMEM supplemented with $1 \mu \mathrm{l}$ of fluorescent dichloro-dihydro-fluorescein diacetate (DCFH-DA; Beyotime Institute of Biotechnology) at $37^{\circ} \mathrm{C}$ for $30 \mathrm{~min}$ in the dark. Cells were washed twice with PBS and fluorescence was observed under an Olympus LCX100 imaging system (Olympus Corporation). The average fluorescence intensity was measured using ImageJ v1.8.0.112 software (National Institutes of Health).

Cell viability assay. Following treatment with LPS, Cell Counting Kit-8 (CCK-8) reagent (APExBIO Technology LLC) was added to a 96-well plate and $\mathrm{H} 9 \mathrm{c} 2$ cells were incubated at $37^{\circ} \mathrm{C}$ for $4 \mathrm{~h}$ in the dark. Absorbance was measured using an enzyme-labeled instrument (BioTek Instruments, Inc.), at a wavelength of $450 \mathrm{~nm}$. The average optical density (OD) was used to calculate cell viability using the following equation: Cell viability $=($ experimental group OD - blank control group OD)/(normal control group OD - blank control group OD) $\times 100 \%$.

Lactate dehydrogenase ( $L D H$ ) cytotoxicity assay. Following treatment with LPS, the cell culture medium was collected and centrifuged at $11,588 \mathrm{x}$ g for $15 \mathrm{~min}$ at $4^{\circ} \mathrm{C}$, and the supernatant was collected. LDH activity was measured to evaluate the damage status of H9c2 cells using Lactate dehydrogenase assay kit (Nanjing Jiancheng Bioengineering Institute), according to the manufacturer's instructions. Absorbance was measured at a wavelength of $440 \mathrm{~nm}$ using an enzyme-labeled instrument (BioTek Instruments, Inc.). LDH activity was calculated according to the manufacturer's instructions.

Propidium iodide (PI) staining. Following treatment with LPS, the culture medium was removed and cells were washed twice with PBS. PI (Beyotime Institute of Biotechnology) was subsequently added to each culture dish, according to the manufacturer's instructions. H9c2 cells were stained with PI at $37^{\circ} \mathrm{C}$ for $30 \mathrm{~min}$. Fluorescent images of the cells were captured using an Olympus LCX100 imaging system. The average fluorescence intensity was measured using ImageJ v1.8.0.112 software (National Institutes of Health).

Statistical analysis. Statistical analysis was performed using GraphPad Pro Prism 8.0 software (GraphPad Software, Inc.). All experiments were performed in triplicate and data are presented as the mean \pm SEM. Unpaired Student's t-test was used to compare differences between two groups, while one-way ANOVA followed by Tukey's post hoc test were used to compare differences between multiple groups. $\mathrm{P}<0.05$ was considered to indicate a statistically significant difference.

\section{Results}

DDX3X expression in LPS-treated H9c2 cardiomyocytes. RT-qPCR and western blot analyses were performed to detect DDX3X expression in H9c2 cells treated with different concentrations of LPS $(0,100,500$ and $1,000 \mathrm{ng} / \mathrm{ml})$. The results demonstrated that the mRNA and protein expression levels of DDX3X increased following treatment with LPS for $24 \mathrm{~h}$, with DDX3X mRNA levels significantly increased at LPS $500(\mathrm{P}<0.01)$ and $1,000 \mathrm{ng} / \mathrm{ml}(\mathrm{P}<0.001$; Fig. $1 \mathrm{~A}-\mathrm{C})$. Taken together, upregulated DDX3X expression in LPS-treated cardiomyocytes suggests that DDX3X participates in the development of LPS-induced cardiomyocyte injury.

LPS induces pyroptosis by increasing intracellular ROS levels and activating the NLRP3 inflammasome. To determine whether LPS provides a priming signal to activate the NLRP3 inflammasome in H9c2 cardiomyocytes, the present study detected the expression levels of NLRP3 and pro-IL-1 $\beta$. The results demonstrated that the protein and mRNA expression levels of NLRP3 and pro-IL-1 $\beta$ increased following treatment with LPS for $24 \mathrm{~h}$, with NLRP 3 mRNA levels significantly increased at LPS 100, 500 and 1,000 ng/ml (all $\mathrm{P}<0.001$ ), and pro-IL-1 $\beta$ mRNA levels significantly increased at LPS $500 \mathrm{ng} / \mathrm{ml}(\mathrm{P}<0.05)$ and $1,000 \mathrm{ng} / \mathrm{ml}(\mathrm{P}<0.001$; Fig. 2A-D $)$. The present results suggested that LPS acted as a priming signal to promote the expression levels of NLRP3 and pro-IL-1 $\beta$ during NLRP3 inflammasome activation in H9c2 cardiomyocytes. It has been reported that ROS can supply activating signals for the activation of the NLRP3 inflammasome (22). Thus, the present study measured ROS levels in LPS-treated cardiomyocytes. As expected, LPS treatment induced the production of ROS, as ROS levels were positively correlated with increasing LPS concentrations (Fig. 2E). The 

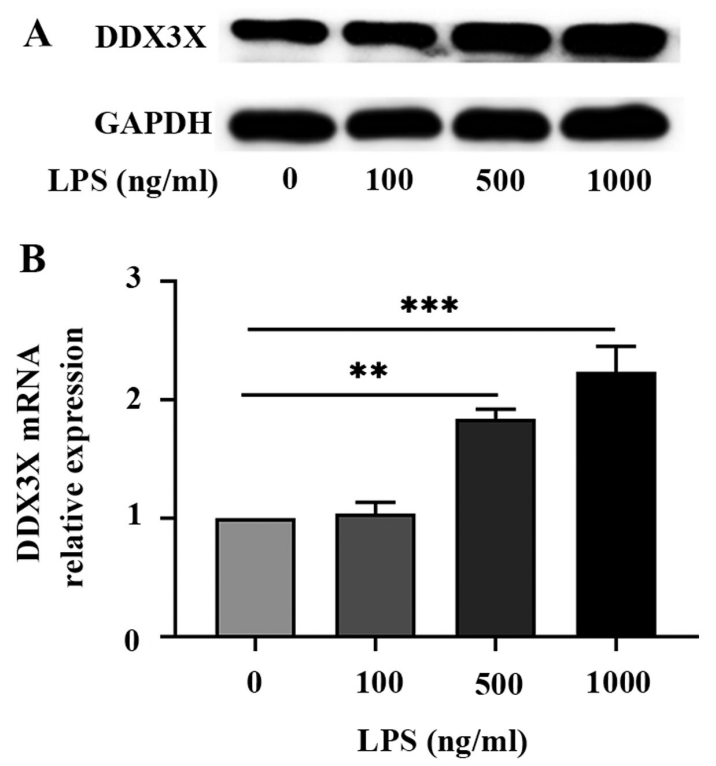

C

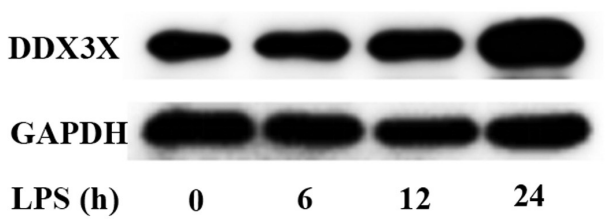

Figure 1. DDX3X expression in LPS-treated cardiomyocytes. (A) Western blot analysis was performed to detect DDX3X protein expression in $\mathrm{H} 9 \mathrm{C} 2$ cardiomyocytes treated with different concentrations of LPS $(0,100$, 500 and $1,000 \mathrm{ng} / \mathrm{ml}$ ) for $24 \mathrm{~h}$. Representative blots (n=3). (B) Reverse transcription-quantitative PCR analysis was performed to detect DDX3X mRNA expression in H9C2 cardiomyocytes treated with different concentrations of LPS $(0,100,500$ and $1,000 \mathrm{ng} / \mathrm{ml})$ for $24 \mathrm{~h}$. Data are presented as the mean \pm SEM $(n=3)$. (C) Western blot analysis was performed to detect DDX3X protein expression in H9C2 cardiomyocytes stimulated with LPS $(1,000 \mathrm{ng} / \mathrm{ml})$ for $0,6,12$ and $24 \mathrm{~h}$. Representative blots $(\mathrm{n}=3) .{ }^{* *} \mathrm{P}<0.01$ and *** $\mathrm{P}<0.001$. LPS, lipopolysaccharide.

protein levels of pro-caspase-1 and caspase-1 p20, as well as caspase-1 activity, were assessed using a caspase- 1 activity assay kit to determine whether LPS treatment can activate a functional NLRP3 inflammasome. As presented in Fig. 2F, the protein expression levels of intracellular pro-caspase-1 and caspase-1 p20 increased in H9c2 cells following treatment with LPS. Cleaved IL-1 $\beta$ was also detected in the supernatant of the cell culture medium. Consistently, the level of cleaved IL-1 $\beta$ notably increased at LPS $1,000 \mathrm{ng} / \mathrm{ml}$ (Fig. 2F). As presented in Fig. 2G, caspase-1 activity significantly increased at LPS $100(\mathrm{P}<0.05), 500(\mathrm{P}<0.01)$ and 1,000 ng/ml $(\mathrm{P}<0.001)$. Collectively, the present results confirm that LPS stimulation activated the NLRP3 inflammasome in $\mathrm{H} 9 \mathrm{c} 2$ cells.

Ddx3x knockdown blocks NLRP3 inflammasome activation in LPS-stimulated H9c2 cardiomyocytes. To understand the regulatory effect of DDX3X in LPS-induced cardiomyocyte pyroptosis and cell injury, H9c2 cardiomyocytes were transfected with $D d x 3 x$ siRNA. Western blot and RT-qPCR analyses confirmed the efficiency of $D d x 3 x$ knockdown (Fig. 3A and B). The present study also compared the levels of intracellular DDX3X, NLRP3 and IL-1 $\beta$, as well as the levels of caspase-1 cleavage and cleaved IL-1 $\beta$ in the supernatant of the culture medium, in $\mathrm{H} 9 \mathrm{c} 2$ cells treated with or without LPS or in the absence or presence of DDX3X. As presented in Fig. 3C and D, in the absence of LPS stimulation, no significant differences in the expression levels of NLRP3 and pro-IL-1 $\beta$, the level of caspase-1 cleavage and the accumulation of cleaved IL-1 $\beta$ were observed between the $D d x 3 x$-silenced and control groups. These results suggest that $D d x 3 x$ knockdown has no influence on the signaling pathway regulating NLRP3 inflammasome activation in the absence of LPS treatment. When $\mathrm{H} 9 \mathrm{c} 2$ cells were stimulated with LPS $1,000 \mathrm{ng} / \mathrm{ml}$, the levels of caspase-1 $(\mathrm{P}<0.01)$ cleavage and cleaved IL-1 $\beta(\mathrm{P}<0.001)$ significantly decreased in DDX3X-deficient cells, while no significant difference in NLRP3 expression was observed between LPS-stimulated cells with normal or altered DDX3X expression (Fig. 3C). Caspase-1 activity also decreased $(\mathrm{P}<0.01)$ following $D d x 3 x$ knockdown in $\mathrm{H} 9 \mathrm{c} 2$ cells treated with LPS $1,000 \mathrm{ng} / \mathrm{ml}$ (Fig. 3E). Taken together, these results suggest that $D d x 3 x$ knockdown blocks NLRP3 inflammasome activation but has no effect on NLRP3 expression in LPS-treated H9c2 cardiomyocytes.

Ddx3x knockdown attenuates pyroptotic cell death and cell injury in LPS-treated H9c2 cardiomyocytes. Cell cytotoxicity was assessed by detecting the levels of released LDH, and cell viability was detected via the CCK-8 assay. $\mathrm{LDH}$ release is a sensitive biomarker of cardiac injury (34); thus, the present study detected the levels of LDH in the supernatant of the culture medium. As presented in Fig. 4A, DDX3X deficiency reversed the increase in LDH level in cells treated with LPS. In addition, cell viability increased following $D d x 3 x$ knockdown in H9c2 cells treated with LPS (Fig. 4B). However, in H9c2 cells not treated with LPS, knockdown of $D d x 3 x$ had no significant effect on cell viability or LDH release. PI staining was performed to detect pyroptotic cell death in H9c2 cells. As expected, $D d x 3 x$ knockdown attenuated pyroptotic cell death in LPS-treated cardiomyocytes (Fig. 4C). Collectively, these results suggest that $D d x 3 x$ knockdown improves the viability of $\mathrm{H} 9 \mathrm{c} 2$ cardiomyocytes treated with LPS. The interactions uncovered by the present results are presented in Fig. 5.

\section{Discussion}

To the best of our knowledge, the present study was the first study to demonstrate that DDX3X is an essential molecular component of the signaling pathway of LPS-induced cardiomyocyte pyroptosis and cell injury. The results demonstrated that DDX3X expression was significantly increased in LPS-treated cardiomyocytes in vitro. Notably, $D d x 3 x$ knockdown attenuated LPS-induced cardiomyocyte pyroptosis and cell injury.

Pyroptosis is a major pathophysiological mechanism in several cardiovascular diseases, such as atherosclerosis, ischemic heart disease, diabetic cardiomyopathy, and cardiac hypertrophy (35). During sepsis, pyroptosis protects the host by eliminating infected cells; however, overactivated pyroptosis can lead to systemic inflammation, resulting in septic shock, MODS and an increased risk of secondary infection (36). The present study detected the upregulation of NLRP3 and pro-IL-1 $\beta$, and the activation of caspase- 1 in $\mathrm{H} 9 \mathrm{c} 2$ cells treated with LPS, demonstrating that LPS stimulation can directly 
Table II. Primer sequences used for quantitative PCR.

\begin{tabular}{llrr}
\hline Gene & \multicolumn{1}{c}{ Sequence } & Product size $(\mathrm{bp})\left(5^{\prime}-3^{\prime}\right)$ & TM $\left({ }^{\circ} \mathrm{C}\right)$ \\
\hline DDX3X-Rat & F: AAACCTTGGTCTTGCCACCTC & 21 & 57.57 \\
NLRP3-Rat & R: CCACGGCTGCTACCCTTATAG & 21 & 59.52 \\
& F GCTAAGAAGGACCAGCCAGA & 20 & 57.45 \\
IL-1 $\beta$-Rat & R: TCCCAGCAAACCTATCCACT & 21 & 55.40 \\
& F: CACCTCTCAAGCAGAGCACAG & 21 & 59.76 \\
GAPDH-Rat & R: GGGTTCCATGGTGAAGTCAAC & 20 & 57.80 \\
& F: GTATTGGGCGCCTGGTCACC & 22 & 61.55 \\
\hline
\end{tabular}

NLRP3, NOD-like receptor protein 3; F, forward; R, reverse; bp, base pair; TM, melting temperature.

A

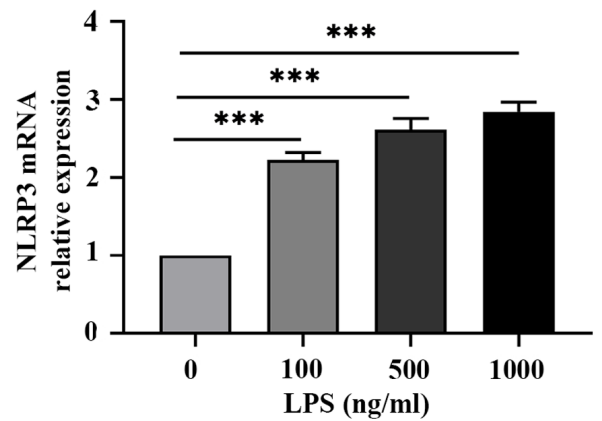

C

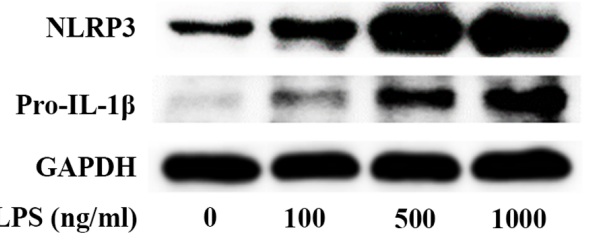

$\mathbf{E}$

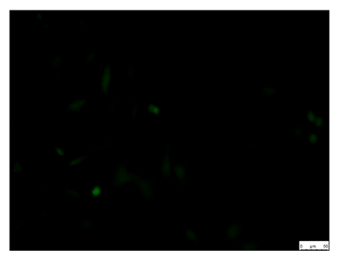

$\operatorname{LPS}(\mathbf{n g} / \mathrm{ml}) \quad 0$

$\mathbf{F}$

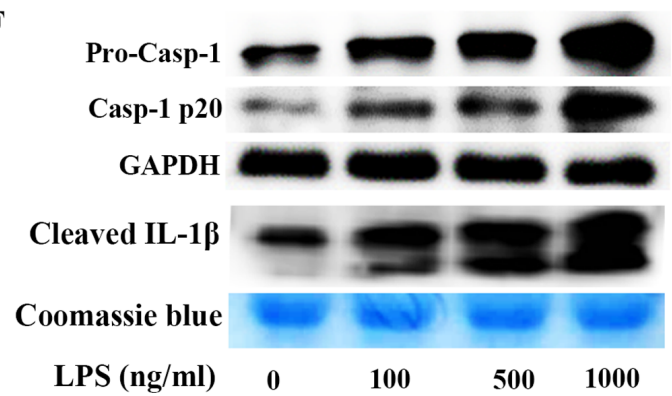

B

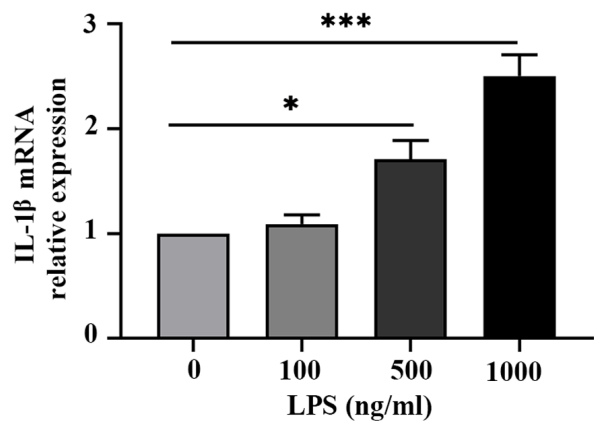

D
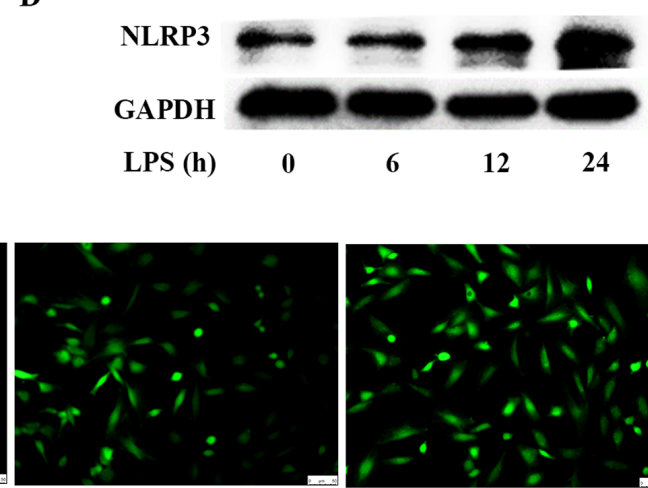

500

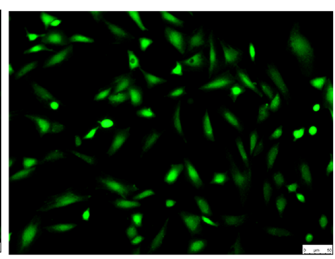

1000

G

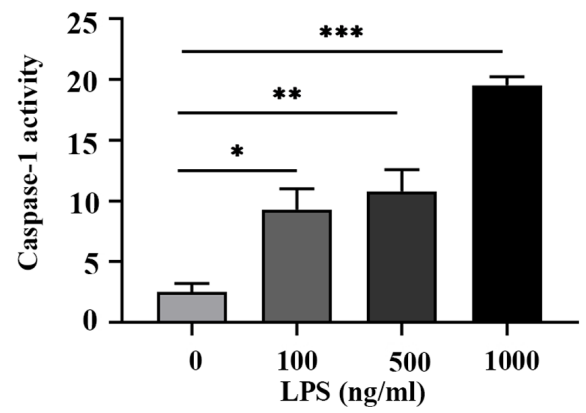

Figure 2.LPS induces pyroptosis by increasing intracellular reactive oxygen species levels and activating NLRP3 inflammasome. Reverse transcription-quantitative PCR analysis was performed to detect the mRNA expression levels of (A) NLRP3 and (B) IL-1 $\beta$ in H9C2 cardiomyocytes treated with different concentrations of LPS $(0,100,500$ and $1,000 \mathrm{ng} / \mathrm{ml})$ for $24 \mathrm{~h}$. Data are presented as the mean $\pm \operatorname{SEM}(\mathrm{n}=4)$. (C) Western blot analysis was performed to detect the protein expression levels of NLRP3 and pro-IL-1 $\beta$ in H9C2 cardiomyocytes treated with different concentrations of LPS $(0,100,500 \mathrm{and} 1,000 \mathrm{ng} / \mathrm{ml})$ for $24 \mathrm{~h}$. Representative blots $(\mathrm{n}=3)$. (D) Western blot analysis was performed to detect NLRP3 protein expression in H9C2 cardiomyocytes stimulated with LPS $(1,000 \mathrm{ng} / \mathrm{ml})$ for $0,6,12$ and $24 \mathrm{~h}$. Representative blots (n=3). (E) The DCFH-DA assay was performed to detect ROS production in H9C2 cardiomyocytes treated with LPS (1,000 ng/ml) for $6 \mathrm{~h}$. $\operatorname{Bar}, 50 \mu \mathrm{m}(\mathrm{n}=3)$. (F) Western blot analysis was performed to detect intracellular protein levels of pro-caspase-1 and caspase-1 p20, and the level of the cleaved IL-1 $\beta$ in the supernatant of culture medium in $\mathrm{H} 9 \mathrm{C} 2$ cardiomyocytes treated with different concentration of LPS $(0,100,500 \mathrm{and} 1,000 \mathrm{ng} / \mathrm{ml})$ for $24 \mathrm{~h}$. Representative blots (n=3). (G) Caspase-1 activity was detected in H9C2 cardiomyocytes treated with different concentrations of LPS (0, 100, $500 \mathrm{and} 1,000 \mathrm{ng} / \mathrm{ml})$ for $24 \mathrm{~h}$. Data are presented as the mean $\pm \operatorname{SEM}(\mathrm{n}=3) .{ }^{*} \mathrm{P}<0.05,{ }^{* *} \mathrm{P}<0.01$ and ${ }^{* * * *} \mathrm{P}<0.001$. LPS, lipopolysaccharide; NLRP3, NOD-like receptor protein3. 
A

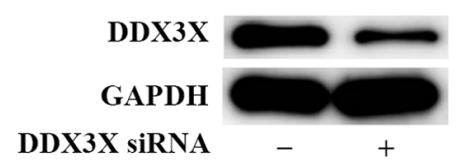

C

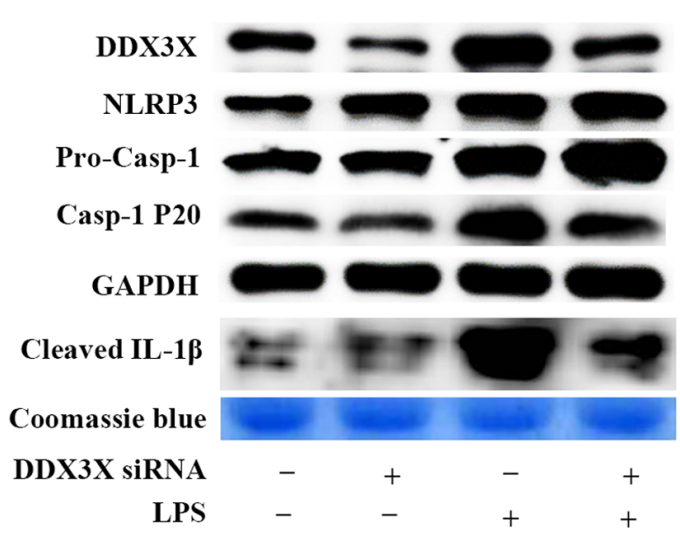

B

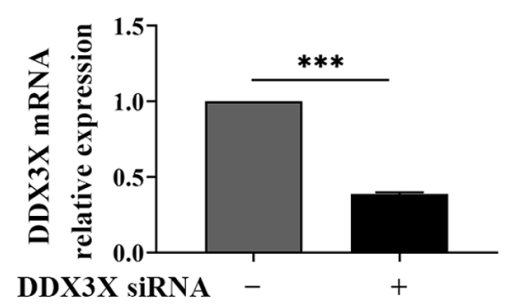

D

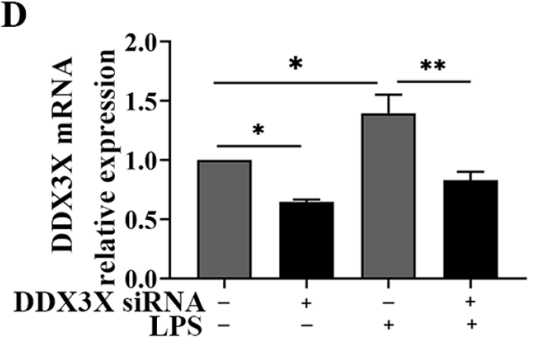

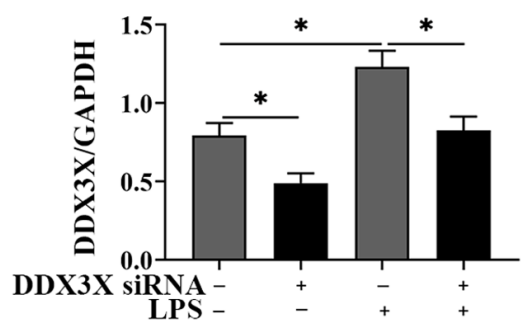

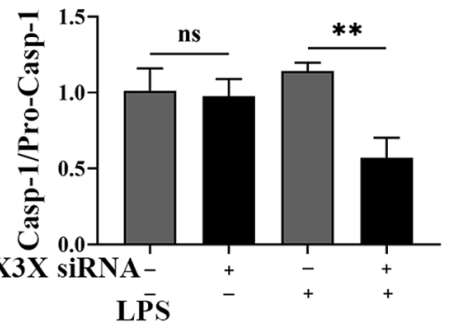

LP̄S
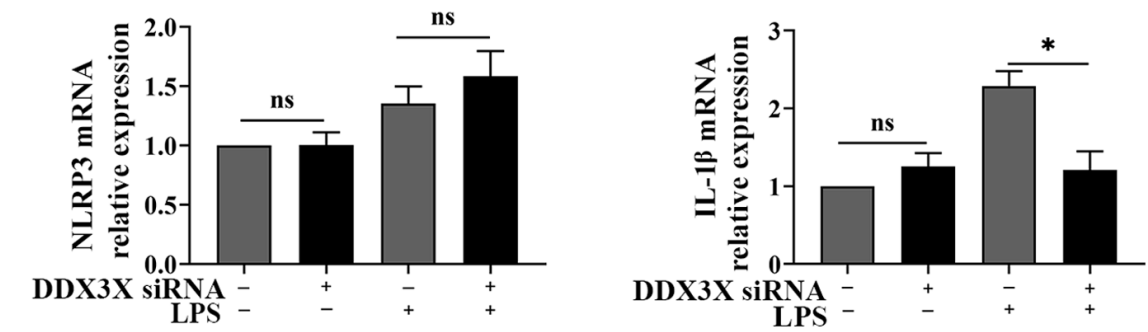

E

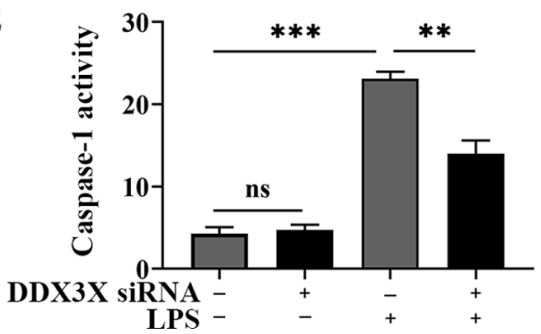

Figure 3. $D d x 3 x$ knockdown blocks NLRP3 inflammasome activation in LPS-stimulated H9C2 cardiomyocytes. (A) Western blotting confirmed the efficiency of $D d x 3 x$ knockdown. Representative blots (n=3). (B) RT-qPCR analysis confirmed the efficiency of $D d x 3 x$ knockdown. Data are presented as the mean \pm SEM $(n=3)$. (C) Western blot analysis was performed to detect the protein expression levels of DDX3X, NLRP3, caspase-1 cleavage and cleaved IL-1 $\beta$ in the supernatant of culture medium in H9C2 cardiomyocytes treated with or without LPS $(1,000 \mathrm{ng} / \mathrm{ml})$ for $24 \mathrm{~h}$ following siRNA-mediated knockdown of $D d x 3 x$. Data are presented as the mean \pm SEM ( $n=3$ ). (D) RT-qPCR analysis was performed to detect the mRNA expression levels of DDX3X, NLRP3 and IL-1 $\beta$ in H9C2 cardiomyocytes treated with or without LPS $(1,000 \mathrm{ng} / \mathrm{ml})$ for $24 \mathrm{~h}$ following siRNA-mediated knockdown of $D d x 3 x$. Data are presented as the mean \pm SEM $(\mathrm{n}=3)$. (E) Caspase-1 activity was detected in H9C2 cardiomyocytes treated with or without LPS $(1,000 \mathrm{ng} / \mathrm{ml})$ for $24 \mathrm{~h}$ following siRNA-mediated knockdown of $D d x 3 x$. Data are presented as the mean \pm SEM $(\mathrm{n}=3) .{ }^{*} \mathrm{P}<0.05,{ }^{* * *} \mathrm{P}<0.01$ and ${ }^{* * * *} \mathrm{P}<0.001$. NLRP3, NOD-like receptor protein 3; LPS, lipopolysaccharide; RT-qPCR, reverse transcription-quantitative PCR; si, small interfering; +, presence; -, absence; ns, not significant.

trigger pyroptosis by activating the NLRP3 inflammasome in H9c2 cardiomyocytes and lead to cardiomyocyte damage.

Increasing evidence suggest that the NLRP3 inflammasome participates in sepsis-induced cardiomyopathy (34,37-39). The NLRP3 inflammasome mediates caspase-1 activation and the excretion of the proinflammatory cytokines, IL-1 $\beta$ and IL-18, resulting in pyroptosis and systemic inflammatory responses. NLRP3 inflammasome activation is essential for normal host defense against microbial infections. However, dysregulated activation of the NLRP3 inflammasome can give rise to severe auto-inflammatory states (37). Thus, activation of the NLRP3 inflammasome must be tightly regulated to provide adequate immune safeguards rather than damage to the host. Several mechanisms have been demonstrated to regulate inflammasome activation, including molecular post-translational modifications of NLRP3 and its interacting partners (21). 
A

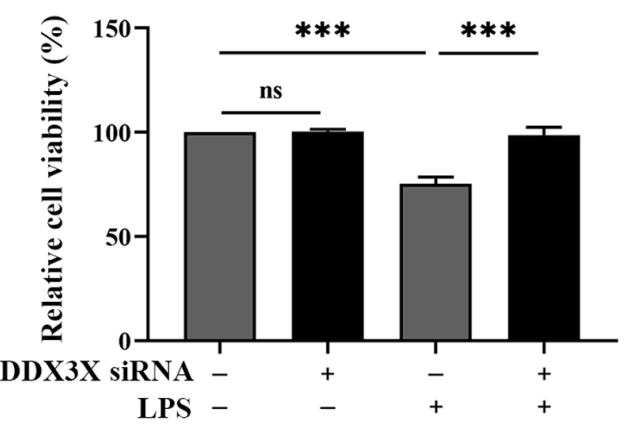

B

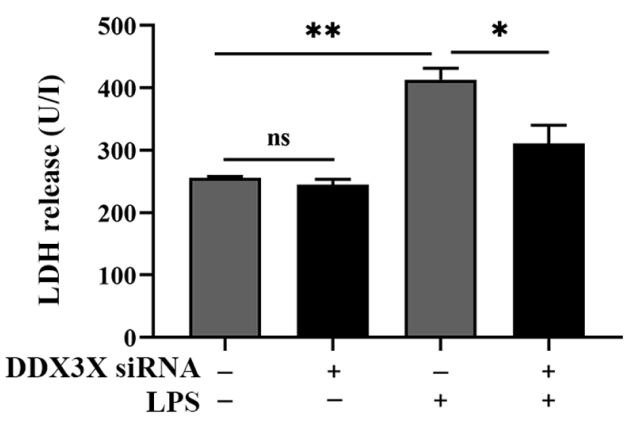

C
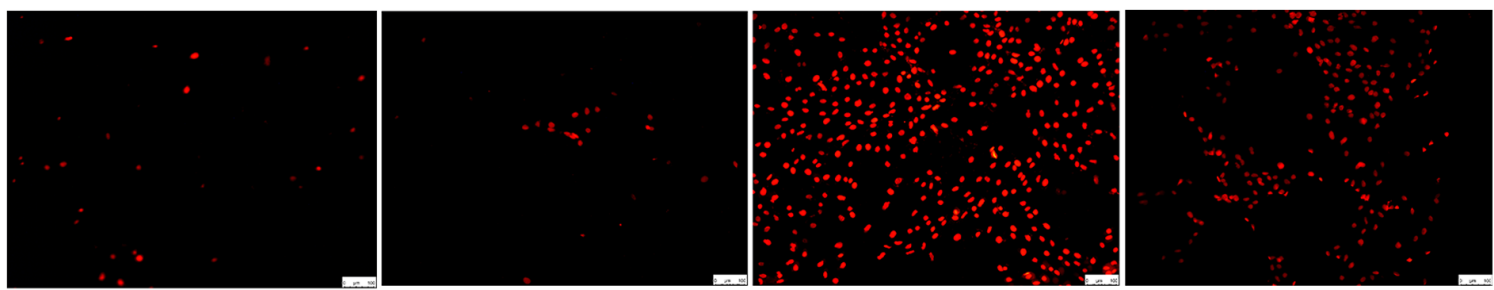

DDX3X SIRNA

LPS

$+$

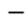

$+$

$-$

Figure 4. $D d x 3 x$ knockdown inhibits NOD-like receptor protein 3 inflammasome activation in LPS-treated cardiomyocytes. (A) Cell Counting Kit-8 assay was performed to detect the viability of $\mathrm{H} 9 \mathrm{C} 2$ cells. Data are presented as the mean \pm SEM $(n=4)$. (B) LDH release was measured via the LDH activity assay. Data are presented as the mean \pm SEM $(n=4)$. (C) The PI staining assay was performed to assess the status of pyroptotic cell death. Bar, $100 \mu \mathrm{m}(\mathrm{n}=3)$. "P<0.05, ${ }^{* *} \mathrm{P}<0.01$ and ${ }^{* * *} \mathrm{P}<0.001$. LPS, lipopolysaccharide; $\mathrm{LDH}$, lactate dehydrogenase; PI, propidium iodide; si, small interfering; ns, not significant.

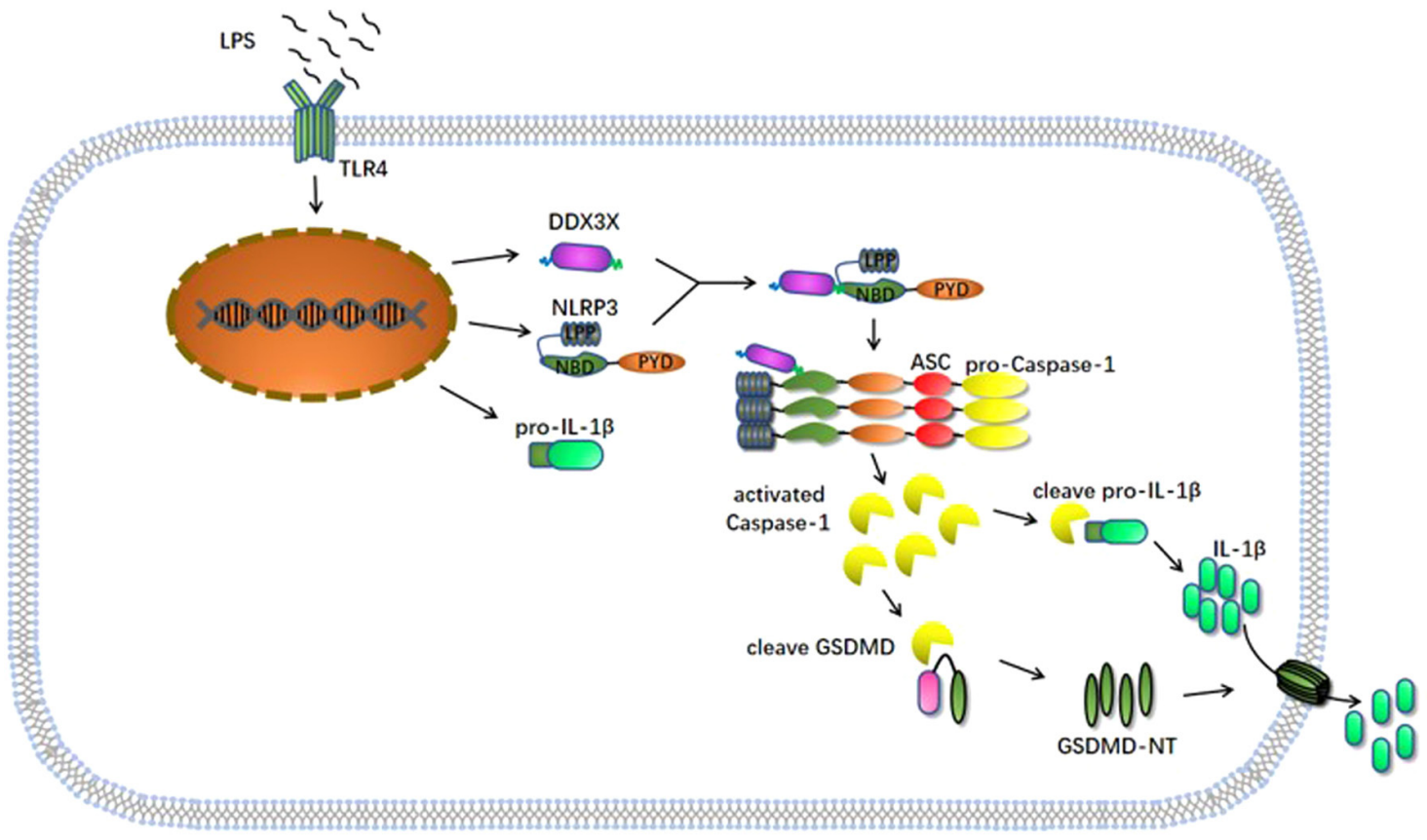

Figure 5. A putative scheme illustrating the mechanism by which DDX3X contributes to pyroptosis of cardiomyocytes in LPS-induced cardiac injury. LPS binding to TLR4 promotes the upregulation of DDX3X, NLRP3 and IL-1 $\beta$. DDX3X directly binds to the NACHT domain of NLRP3, contributing to the NLRP3 inflammasome assembly and the activation of caspase-1. Activated caspase-1 induces the maturation of IL-1 $\beta$ and shears GSDMD. Subsequently, the cleaved N-terminal domain of GSDMD translocates to the plasma membrane and forms pores, facilitating the extracellular secretion of IL-1 $\beta$ into the circulation system and triggers pyroptosis. LPS, lipopolysaccharide; TLR4, Toll-like receptor 4; NLRP3, NOD-like receptor protein 3; GSDMD, gasdermin D.

DDX3X is a functionally multifaceted helicase, which plays various roles in RNA metabolism, cell cycle control, stress granule formation, apoptosis, innate immunity, viral infection and cancer (40). A previous study suggested that the availability of DDX3X molecules regulated pyroptosis mediated by the NLRP3 inflammasome in macrophages, and that DDX3X was involved in NLRP3 inflammasome assembly through direct binding to the NACHT domain of NLRP3 (28). Inhibition of the ATPase activity of DDX3X by RK-33 does not affect NLRP3 inflammasome activation, suggesting 
that DDX3X exerts a scaffold function by facilitating the assembly of ASC specks, rather than a catalytic function (41). In the present study, DDX3X expression in cardiomyocytes increased following treatment with LPS, suggesting that DDX3X is involved in LPS-induced cardiomyocyte stress response. The results also demonstrated that $D d x 3 x$ knockdown inhibited LPS-induced NLRP3 inflammasome activation but did not affect NLRP3 expression. The results further confirmed that DDX3X promotes NLRP3 inflammasome assembly by interacting with NLRP3. The inhibition of NLRP3 inflammasome activation further hindered caspase-1 activation, which attenuated pyroptosis and cell death in LPS-treated cardiomyocytes. Taken together, these results suggest that DDX3X acts as a regulator of pyroptosis during cardiomyocyte stress response.

In conclusion, the results of the present study demonstrated that LPS stimulation induced DDX3X expression in cardiomyocytes, and $D d x 3 x$ knockdown attenuated LPS-induced cardiomyocyte pyroptosis and cell injury by suppressing NLRP3 inflammasome activation. However, no in vivo data were provided in the present study. Thus, further studies are required to confirm whether DDX $3 X$ may be a potential therapeutic target for sepsis-induced cardiomyopathy.

\section{Acknowledgements}

Not applicable.

\section{Funding}

The present study was supported by the National Nature Science Foundation of China (grant no. 81873473), the Academic Promotion Program of Shandong First Medical University (grant no. 2019QL014) and Shandong Taishan Scholarship (no grant number available).

\section{Availability of data and materials}

The datasets used and/or analyzed during the current study are available from the corresponding author on reasonable request.

\section{Authors' contributions}

DF, LG, JiL, HH, JuL and EH were involved in the study design. DF, YS and XM performed the experiments. DF analyzed the data and drafted the initial manuscript. All authors have read and approved the final manuscript. DF and EH confirm the authenticity of all the raw data.

\section{Ethics approval and consent to participate}

Not applicable.

\section{Patient consent for publication}

Not applicable.

\section{Competing interests}

The authors declare that they have no competing interests.

\section{References}

1. Deutschman CS and Tracey KJ: Sepsis: Current dogma and new perspectives. Immunity 40: 463-475, 2014.

2. van Engelen TSR, Wiersinga WJ, Scicluna BP and van der Poll T: biomarkers in sepsis. Crit Care Clin 34: 139-152, 2018.

3. Sato R and Nasu M: A review of sepsis-induced cardiomyopathy. J Intensive Care 3: 48, 2015.

4. Li F, Lang F, Zhang H, Xu L, Wang Y, Zhai C and Hao E: Apigenin alleviates endotoxin-induced myocardial toxicity by modulating inflammation, oxidative stress, and autophagy. Oxid Med Cell Longev 2017: 2302896, 2017.

5. Hao E, Lang F, Chen Y, Zhang H, Cong X, Shen X and Su G: Resveratrol alleviates endotoxin-induced myocardial toxicity via the Nrf2 transcription factor. PLoS One 8: e69452, 2013.

6. Peng F, Chang W, Sun Q, Xu X, Xie J, Qiu H and Yang Y: HGF alleviates septic endothelial injury by inhibiting pyroptosis via the mTOR signalling pathway. Respir Res 21: 215, 2020.

7. Dai XG, Li Q, Li T, Huang WB, Zeng ZH, Yang Y, Duan ZP, Wang YJ and Ai YH: The interaction between C/EBP $\beta$ and TFAM promotes acute kidney injury via regulating NLRP3 inflammasome-mediated pyroptosis. Mol Immunol 127: 136-145, 2020.

8. Fu Q, Wu J, Zhou XY, Ji MH, Mao QH, Li Q, Zong MM, Zhou ZQ and Yang JJ: NLRP3/caspase-1 pathway-induced pyroptosis mediated cognitive deficits in a mouse model of sepsis-associated encephalopathy. Inflammation 42: 306-318, 2019.

9. Wu C, Lu W, Zhang Y, Zhang G, Shi X, Hisada Y, Grover SP, Zhang X, Li L, Xiang B, et al: Inflammasome activation triggers blood clotting and host death through pyroptosis. Immunity 50: 1401-1411.e4, 2019.

10. Bordon Y: Mucosal immunology: Inflammasomes induce sepsis following community breakdown. Nat Rev Immunol 12: 400-401, 2012.

11. Pu Q, Gan C, Li R, Li Y, Tan S, Li X, Wei Y, Lan L, Deng X, Liang H, Ma F and Wu M: Atg7 deficiency intensifies inflammasome activation and pyroptosis in pseudomonas sepsis J Immunol 198: 3205-3213, 2017.

12. Li Z, Guo J and Bi, L: Role of the NLRP3 inflammasome in autoimmune diseases. Biomed Pharmacother 130, 110542, 2020.

13. Martinon F, Burns K and Tschopp J: The inflammasome: A molecular platform triggering activation of inflammatory caspases and processing of proIL-beta. Mol Cell 10: 417-426, 2002.

14. Kanneganti TD, Ozören N, Body-Malapel M, Amer A, Park JH, Franchi L, Whitfield J, Barchet W, Colonna M, Vandenabeele P, et al: Bacterial RNA and small antiviral compounds activate caspase-1 through cryopyrin/Nalp3. Nature 440: 233-236, 2006.

15. Mariathasan S, Weiss DS, Newton K, McBride J, O'Rourke K, Roose-Girma M, Lee WP, Weinrauch Y, Monack DM and Dixit VM: Cryopyrin activates the inflammasome in response to toxins and ATP. Nature 440: 228-232, 2006.

16. Karki R and Kanneganti TD: Diverging inflammasome signals in tumorigenesis and potential targeting. Nat Rev Cancer 19: 197-214, 2019.

17. Malireddi RKS, Gurung P, Mavuluri J, Dasari TK, Klco JM, Chi $\mathrm{H}$ and Kanneganti TD: TAK1 restricts spontaneous NLRP3 activation and cell death to control myeloid proliferation. J Exp Med 215: 1023-1034, 2018.

18. Man SM and Kanneganti TD: Converging roles of caspases in inflammasome activation, cell death and innate immunity. Nat Rev Immunol 16: 7-21, 2016.

19. Venegas C, Kumar S, Franklin BS, Dierkes T, Brinkschulte R, Tejera D, Vieira-Saecker A, Schwartz S, Santarelli F, Kummer MP, et al: Microglia-derived ASC specks cross-seed amyloid- $\beta$ in Alzheimer's disease. Nature 552: 355-361, 2017.

20. He Y, Hara H and Núñez G: Mechanism and regulation of NLRP3 inflammasome activation. Trends Biochem Sci 41: 1012-1021, 2016.

21. Kelley N,JeltemaD,Duan Y and He Y: The NLRP3 Inflammasome: An overview of mechanisms of activation and regulation. Int J Mol Sci 20: 3328, 2019.

22. Tschopp J and Schroder K: NLRP3 inflammasome activation: The convergence of multiple signalling pathways on ROS production? Nat Rev Immunol 10: 210-215, 2010.

23. Zhou R, Tardivel A, Thorens B, Choi I and Tschopp J: Thioredoxininteracting protein links oxidative stress to inflammasome activation. Nat Immunol 11: 136-140, 2010.

24. Beckham C, Hilliker A, Cziko AM, Noueiry A, Ramaswami M and Parker R: The DEAD-box RNA helicase Ded1p affects and accumulates in Saccharomyces cerevisiae P-bodies. Mol Biol Cell 19: 984-993, 2008. 
25. Garbelli A, Radi M, Falchi F, Beermann S, Zanoli S, Manetti F, Dietrich U, Botta M and Maga G: Targeting the human DEAD-box polypeptide 3 (DDX3) RNA helicase as a novel strategy to inhibit viral replication. Curr Med Chem 18: 3015-3027, 2011.

26. Soulat D, Bürckstümmer T, Westermayer S, Goncalves A, Bauch A, Stefanovic A, Hantschel O, Bennett KL, Decker T and Superti-Furga G: The DEAD-box helicase DDX3X is a critical component of the TANK-binding kinase 1-dependent innate immune response. EMBO J 27: 2135-2146, 2008.

27. Botlagunta M, Vesuna F, Mironchik Y, Raman A, Lisok A, Winnard P Jr, Mukadam S, Van Diest P, Chen JH,Farabaugh P, et al: Oncogenic role of DDX3 in breast cancer biogenesis. Oncogene 27 3912-3922, 2008

28. Bol GM, Vesuna F, Xie M, Zeng J, Aziz K, Gandhi N, Levine A, Irving A, Korz D, Tantravedi S, et al: Targeting DDX3 with a small molecule inhibitor for lung cancer therapy. EMBO Mol Med 7: 648-669, 2015.

29. Samir P, Kesavardhana S, Patmore DM, Gingras S, Malireddi RK, Karki R, Guy CS, Briard B, Place DE, Bhattacharya A, et al: DDX 3X acts as a live-or-die checkpoint in stressed cells by regulating NLRP3 inflammasome. Nature 573: 590-594, 2019.

30. Lu A, Magupalli VG, Ruan J, Yin Q, Atianand MK, Vos MR, Schröder GF, Fitzgerald KA, Wu H and Egelman EH: Unified polymerization mechanism for the assembly of ASC-dependent inflammasomes. Cell 156: 1193-1206, 2014.

31. Franklin BS, BossallerL, DeNardoD, Ratter JM,Stutz A,Engels G, Brenker C, Nordhoff M, Mirandola SR, Al-Amoudi A, et al: The adaptor ASC has extracellular and 'prionoid' activities that propagate inflammation. Nat Immunol 15: 727-737, 2014.

32. Duncan JA, Bergstralh DT, Wang Y, Willingham SB, Ye Z, Zimmermann AG and Ting JP: Cryopyrin/NALP3 binds ATP/dATP, is an ATPase, and requires ATP binding to mediate inflammatory signaling. Proc Natl Acad Sci USA 104: 8041-8046, 2007.

33. Mavridis K, Stravodimos K and Scorilas A: Downregulation and prognostic performance of microRNA 224 expression in prostate cancer. Clin Chem 59: 261-269, 2013.
34. Li N, Zhou H, Wu H, Wu Q, Duan M, Deng W and Tang Q: STING-IRF3 contributes to lipopolysaccharide-induced cardiac dysfunction, inflammation, apoptosis and pyroptosis by activating NLRP3. Redox Biol 24: 101215, 2019.

35. Zhaolin Z, Guohua L, Shiyuan W and Zuo W: Role of pyroptosis in cardiovascular disease. Cell Prolif 52: e12563, 2019.

36. Gao YL, Zhai JH and Chai YF: Recent advances in the molecular mechanisms underlying pyroptosis in sepsis. Mediators Inflamm 2018: 5823823, 2018.

37. Kalbitz M, Fattahi F, Grailer JJ, Jajou L, Malan EA, Zetoune FS, Huber-Lang M, Russell MW and Ward PA: Complement-induced activation of the cardiac NLRP3 inflammasome in sepsis. FASEB J 30: 3997-4006, 2016.

38. Zhang W, Tao A, Lan T, Cepinskas G, Kao R, Martin CM and Rui T: Carbon monoxide releasing molecule-3 improves myocardial function in mice with sepsis by inhibiting NLRP3 inflammasome activation in cardiac fibroblasts. Basic Res Cardiol 112: 16, 2017.

39. Guo T, Jiang ZB, Tong ZY, Zhou Y, Chai XP and Xiao XZ: Shikonin Ameliorates LPS-induced cardiac dysfunction by SIRT1-dependent inhibition of NLRP3 inflammasome. Front Physiol 11: 570441, 2020.

40. Mo J, Liang H, Su C, Li P, Chen J and Zhang B: DDX3X: Structure, physiologic functions and cancer. Mol Cancer 20: 38, 2021.

41. Samir P and Kanneganti TD: DDX3X Sits at the crossroads of liquid-liquid and prionoid phase transitions arbitrating life and death cell fate decisions in stressed cells. DNA Cell Biol 39: 1091-1095, 2020.

cc) (i) $\ominus$ This work is licensed under a Creative Commons

(c) Attribution-NonCommercial-NoDerivatives 4.0 International (CC BY-NC-ND 4.0) License. 